\title{
Conjugated Linoleic Acid (Cla) Intake, A Mini Review
}

\author{
Martha Gabriela Campos Mondragón ${ }^{1}$ \\ ${ }^{I}$ (Veracruzan University. Nutrition Faculty Campus Veracruz)
}

\begin{abstract}
Conjugated linoleic acid or CLA, is among the bioactive components which are interesting for obesity treatment. It is a term that includes a conjugated isomer group of the linoleic acid, of which two of them cis-9, trans-11 and trans-10, cis-12 are capable of reducing body fat in animals and human beings. The best source of CLA in food is found in meat and dairy, which come from ruminants. The anti-obesity effect of the CLA has been demonstrated by the reduction on the indicators of the obesity, as body fat and waist circumference. Although the effect of CLA on the reduction of body fat is well known, it is important to make a distinction in the condition of patients and the consumed dose because CLA also has modified other cardiovascular risk markers. While the CLA intake has showed a positive effect and less cardiovascular related risk factors, also has increased the insulin resistance in type 2 diabetes mellitus patients. Therefore, the purpose of the present text was to review the most important aspects about the CLA intake in humans, like the dietary sources, the action mechanism and the clinical results.
\end{abstract}

Keywords: obesity, isomers, linoleic acid, diet, health

\section{Introduction}

Obesity is an increasing public health problem and a worldwide phenomenon currently, in such a way that the study of bioactive compounds for its treatment has achieved greater importance. Among these compounds, the conjugated linoleic acid (CLA) has demonstrated to reduce the body fat. The CLA is synthesized by a natural way in the ruminant metabolism. As a result, the dietary CLA is consumed naturally and mainly through meat and dairy in humans. Though the term CLA refers to a group of fatty acids, only two have shown biological activity cis-9, trans-11 and trans-10, cis-12. Therefore, the supplementation of the two isomers has been studied in overweight, obese and chronically ill patients.

\section{Linoleic acid}

The linoleic acid (18:2) belongs to the chemical family of the omega six fatty acids (n6). It is indispensable in the human diet. That's because the human cells don't have the desaturase enzymes that removes two hydrogen atoms from a fatty acid, creating a carbon/carbon double bond in the atoms of carbon 12 and carbon 15 ( $\Delta 12$-desaturase and $\Delta 15$-desaturase). Therefore, it is necessary to consume it in the food. It is found in the most of the eatable oil, like safflower, sunflower, corn and soybean.

\section{Conjugated Linoleic Acid (CLA)}

The term conjugated linoleic acid or CLA describes a group of conjugated isomers from linoleic acid. These isomers have two double links in their carbon chain in a conjugated manner, instead of the usual separation for a methylene. The double links could be in trans or cis configuration, which explains the wide spectrum of isomers with variability among them in position $(7,9 ; 8,10 ; 9,11 ; 10,12$ or 11,13$)$ and geometry (cis, cis; cis, trans; trans, trans or trans, cis) ${ }^{\mathbf{1}}$.

Nevertheless, there are two isomers with more known biological activity: cis-9, trans-11 and trans-10, cis-12. The first positive effect identified in CLA was as anti-mutagenic factor in the meat ${ }^{2}$. Later scientific arguments were supported by evidence, about its body fat loss effect in animals and human beings ${ }^{\mathbf{3}, 4}$.

The compound known as CLA is an intermediate of the ruminal bacterial biohydrogenation from linoleic acid (cis-9, cis-12 C 18:2) to stearic acid (C 18:0) by Butyrivibrio fibrisolvens, or by endogenous conversion of the trans vaccenic acid (trans-11 C, 18:1 TVA) by the $\Delta 9$ desaturase enzyme in the mammary gland $^{5}$.

\subsection{Dietary CLA}

The best source of CLA in food is found in meat and dairy, which come from ruminants. It is well known that the fatty acids amount and composition of the bovine milk changes according to the food, period of lactation, seasonal variation, geographical area, presence of mastitis, race, among other factors. The isomers cis9, trans-11 and trans-10, cis-12, are generally in relations of 30:1 to 70:1 in the food ${ }^{6}$. For food of animal origin the amount of the isomer cis-9, trans-11 varies about $75-90 \%$ of the CLA, and in vegetable oils, it is less than $50 \%$. The Table 1 shows the content of CLA in different foods ${ }^{7,8}$. 
Table 1. Foods with important content of CLA

\begin{tabular}{|l|l|}
\hline Food & $\begin{array}{l}\text { CLA } \\
\text { mg/g of fat }\end{array}$ \\
\hline Whole milk & $5.0-7.0$ \\
\hline Lamb & 5.6 \\
\hline Processed cheeses & 5.0 \\
\hline Beaf & $3.0-4.0$ \\
\hline Veal & 2.7 \\
\hline Chicken & 0.9 \\
\hline Fish & $0.1-0.8$ \\
\hline Sunflower oil & 0.7 \\
\hline Seafood & $0.3-0.6$ \\
\hline Coconut oil & 0.1 \\
\hline
\end{tabular}

In 2008, the American Heart Association suggested the consumption of CLA inside the trans lipid group, and established that its ingestion must be less than $1 \%$ of total daily calories. Though CLA doesn't cause the same negative effects than the trans hydrogenated lipids on the blood cholesterol levels, its natural food sources are higher in saturated fatty acids, so it is recommended to reduce its intake. CLA intake depends on the habits from each country. Also affects the CLA percentage given by the meats of ruminant animals and incorporated into a diet. Estimated human intake of CLA has been reported since 0.35-0.43 g/day in Germany up to $1.5-1.8 \mathrm{~g} /$ day in Australia ${ }^{9,10}$.

\subsection{Action of the CLA in the Reduction of the Obesity}

On the basis of animal studies, the following mechanisms of action of CLA have been proposed (Figure 1):

A) CLA increases the energy expenditure because of it reduces the body fat ${ }^{11}$.

B) CLA reduces the fat cell mass or the number of them because of it reduces the body fat. This could happen in four possible ways ${ }^{\mathbf{1 2}}$ : via the lipoprotein lipase inhibition in the adipose cells, by the inhibition of the stearoylCoA desaturase activity, through increasing the apoptosis of the pre adipocytes and adipocytes, and by modulation of lipolysis.

C) CLA reduces the body fat by means of modulation of adipocytokines and cytokines. A study in murine models reported that it increases the adiponectin level and diminishes the TNF- $\alpha$ concentration ${ }^{\mathbf{1 3}}$.

D) CLA increases the fatty acid $\beta$-oxidation in the skeletal muscle. It increases the carnitine palmitoyltransferase type I activity and expression, the rate limiting enzyme in the metabolic pathway ${ }^{\mathbf{1 3}}$.

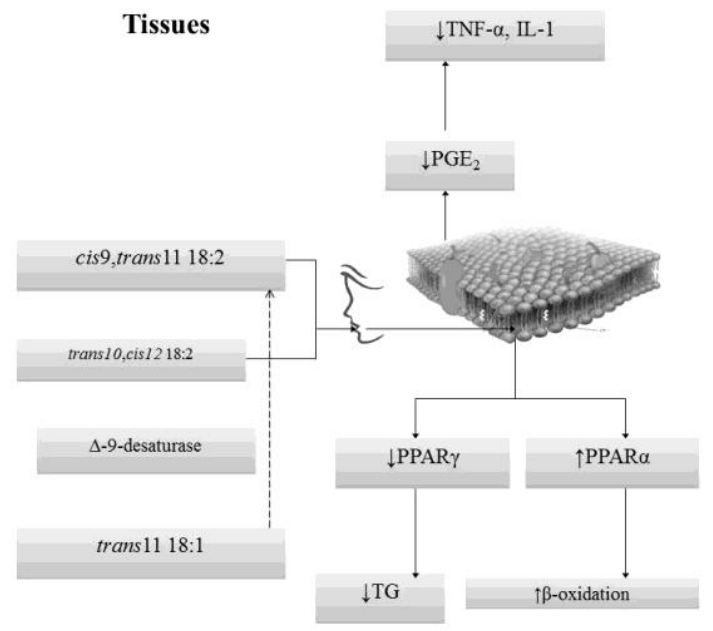

Fig 1. Action mechanism of CLA

In addition, current evidence shows that the isomer trans 10 , cis 12 treatment reduces the adipogenesis and the lipogenesis attenuating the transcription factor expression. Among these transcription factors are the Peroxisome Proliferator-Activated Receptor gamma (PPAR $\gamma$ ), CCAAT-enhancer-binding protein gamma $(\mathrm{C} / \mathrm{EBP} \gamma)$, Sterol regulatory element-binding protein 1c (SREBP-1c), liver X receptor alpha (LXR $\alpha$ ), and AP-2 family of transcription factors ${ }^{\mathbf{1 4 , 1 5}}$. The dietary CLA incorporated into the human cellular membranes modifies their chemical properties. As a consequence, it diminishes the inflammation by means of lesser synthesis of prostaglandin E2 (PGE2), and lesser blood levels of tumor necrosis factor-alpha (TNF- $\alpha$ ) and interleukin-1 (IL1). As well, by means of transcription factor modulation (PPAR), it reduces the synthesis of triacylglycerols (TG) and increases the $\beta$-oxidation of lipids 


\subsection{Modification of Clinical Parameters}

The anti-obesity effect of the CLA has been demonstrated by the reduction on the indicators of the obesity, as body fat and waist circumference. A study in patients with overweight and obesity, tried a 50:50 mixture of isomers cis-9, trans-11 and trans-10, cis-12, in different doses (1.7, 3.4, 5.1 and $6.8 \mathrm{~g} / \mathrm{day})$, all of them reduced the corporal fat, but those with doses higher than $3.4 \mathrm{~g}$ did not produce additional effects ${ }^{4}$. Furthermore, the waist circumference was reduced in obese patients after a CLA consumption of $4.2 \mathrm{~g} / \mathrm{day}$ during 4 weeks, while the weight, body fat and blood insulin, glucose and cholesterol-LDL levels did not show any change ${ }^{16}$.

In overweight patients, the CLA intake of $3.4 \mathrm{~g} /$ day (50:50 rate) during one year reduced body fat and serum cholesterol and leptin levels. Nevertheless, the serum insulin was also increased ${ }^{17}$.

The CLA intake of $3 \mathrm{~g} /$ day (50:50) during 8 weeks in type 2 diabetes mellitus patients, increased the blood HDL-cholesterol level, and diminished the blood fibrinogen level. Though these results showed a positive effect and less cardiovascular related risk factor, this dose of these kinds of patients also showed adverse effects on the insulin and glucose metabolism. That's because of the fact that the CLA increased the fasting blood glucose level and reduced the insulin sensitivity ${ }^{18}$.

Iwata et al (2007) studied the effect of CLA on the transaminases ALT and AST levels. Their results showed that the higher dose (6.8 g CLA, 50:50 during 12 weeks) increased the blood transaminases level, while the lesser dose (3.4 g CLA, 50:50 during 12 weeks) did not affect the hepatic enzymes, which suggested that the last one is a safe dose of the diet of Japanese people with overweight.

A recent review highlights the need for more precise and well-designed long-term intervention studies with controlled food intake and activity level in order to assess the effectiveness of CLA on human health ${ }^{20}$.

\section{Conclusion}

Although the human being can get linoleic acid from the diet, the amounts in the natural foods are too small in relation with the doses that have shown to reduce the body fat. The American Heart Association encourages reducing the CLA intake up to $1 \%$ of the calories of the day in order to not exceed the oily food from which it comes. This means that in an average adult diet of 2000 calories, 20 of them will be coming from the CLA, an amount that could be found in only two grams of meat or dairy fat. A fourth of a cup of whole milk contains two grams of fat with a CLA amount between 10 and $14 \mathrm{mg}$. Therefore, it has been intended the CLA supplementation as an alternative to accomplish amounts higher than 1.7 grams/day, the minimal dose that has been shown effective in diminishing the body fat in human beings.

The knowledge about the action mechanism of the CLA has evolved together with the advances in Nutrigenomics. With the CLA intake, this one joins to the cellular membranes and it is incorporated into phospholipids and then become part of them. Then, the CLA influence the chemical and biological properties of the cellular membranes, such as its fluency, permeability, transmission of signs and regulation of transcription factors. Therefore, CLA intake decreases lipogenic enzyme gene expression and increases the $\beta$-oxidation enzyme gene expression.

The effect of CLA has been assessed in other cardiovascular risk indicators, which are the consequence of the obesity associated complications and its comorbidities. The CLA intake has shown to increase the insulin resistance in type 2 diabetes mellitus patients. If the supplementation period is as short as four weeks, neither the blood lipid levels nor blood glucose levels alters adversely, nevertheless, if the trial period is higher, up one year, the serum insulin level would be increased. Additionally, amounts of 6.8 CLA grams have shown a pro inflammatory effect in the liver, evidenced in the high blood transaminases level. Finally, although there is some evidence about the effect of the CLA on the obesity, the study of CLA intake in humans must be continued in order to establish its safety and efficacy.

\section{References}

[1] J Martin, and F. Valeille, Conjugated linoleic acids: all the same or to everyone its own function?, Reproduction Nutrition Development, 42(6), 2002, 525-536.

[2] Y Ha, N. Grimm, and M. Pariza, Anticarcinogens from fried ground beef: Heat-altered derivatives of linoleic acid, Carcinogenesis, 8, 1987, 1881-1887.

[3] J Delany, F. Blohm, A. Truett, J. Scimeca, and D. West, Conjugated linoleic acid rapidly reduces body fat content in mice without affecting energy intake, American Journal of Physiology, 276, 1999, 1172-1179.

[4] H. Blankson, J. Stakkestad, H. Erling, T. Wadstei, and O. Gudmundsen, Conjugated linoleic acid reduces body fat mass in overweight and obese humans, Journal of Nutrition, 130, 2000, 2943-2948.

[5] B. Corl, L.Baumgard, D. Dwyer, J. Griinari, B. Phillips, and D. Bauman, The role of delta(9)-desaturase in the production of cis-9, trans-11 CLA, The Journal of Nutritional Biochemistry, 12, 2001, 622-630.

[6] E. Poole, and C. McQueen, Conjugated linoleic acid, American Journal of Health-System Pharmacy, 63, 2006, $2056-2062$.

[7] S. Chin, W. Liu, J. Storkson, Y. Ha, and M. Pariza, Dietary sources of conjugated dienoic isomers of linoleic acid, a newly recognized class of anticarcinogens, Journal of Food Composition and Analysis, 5(3), 1992, 185-197.

[8] H. Lin, D. Boylston, J. Chang, O. Luedecke, and D. Shultz, Survey of the Conjugated Linoleic Acid Contents of Dairy Products, Journal of Dairy Science, 78(11), 1995, 2358-2365. 
[9] P. Parodi, Conjugated linoleic acid content: An anticarcinogenic fatty acid present in milk fat, Australian Journal of Dairy Technology, 49, 1994, 93-97.

[10] J. Fritsche, and H. Steinhart, Amounts of conjugated linoleic acid (CLA) in German foods and evaluation of daily intake, Z. J. Zeitschrift für Lebensmitteluntersuchung und-Forschung A. 206, 1998, 1431-4630.

[11] A. Terpstra, Differences between humans and mice in efficacy of the body fat lowering effect of conjugated linoleic acid: role of metabolic rate, Journal of Nutrition, 131, 2001, 2067-2068.

[12] Y. Park, and W. Pariza, Mechanisms of body fat modulation by conjugated linoleic acid CLA, Food Research International, 40, 2007, 311-323.

[13] K. Nagao, N. Inoue, Y. Wang, B. Shirouchi, and T. Yanagita, Dietary conjugated linoleic acid alleviates nonalcoholic fatty liver disease in Zucker (fa/fa) rats, Journal of Nutrition, 135, 2005, 9-13.

[14] K. Kang, W. Liu, K. Albright, Y. Park, and M. Pariza, Trans-10,cis-12 CLA inhibits differentiation of 3T3-L1 adipocytes and decreases PPAR gamma expression, Biochemical and Biophysical Research Communications, 303, $2003,795-799$.

[15] P. La Rosa, J. Riethoven, H. Chen, J. Miner, and M. Fromm, Trans-10, cis-12 conjugated linoleic acid activates the integrated stress response pathway in adipocytes, Physiological Genomics, 31, 2007, 544-553.

[16] U. Riserus, L. Berglund, and B. Vessby, Conjugated linoleic acid (CLA) reduced abdominal adipose tissue in obese middle-aged men with signs of the metabolic syndrome: a randomized controlled trial, International Journal of Obesity, 25, 2001, $1129-1135$.

[17] J.M. Gaullier, J. Halse, K. Høye, K. Kristiansen, H. Fagertun, H. Vik, and O. Gudmundsen, Supplementation with conjugated linoleic acid for 24 months is well tolerated by and reduces body fat mass in healthy, overweight humans, Journal of Nutrition, 135(4), 2005, 778-784.

[18] F. Moloney, T. Yeow, A. Mullen, J. Nolan, and H. Roche, Conjugated linoleic acid supplementation, insulin sensitivity, and lipoprotein metabolism in patients with type 2 diabetes mellitus, American Journal of Clinical Nutrition, 80, 2004, 887-895.

[19] T. Iwata, T. Kamegai, Y. Yamauchi-Sato, A. Ogawa, M. Kasai, T. Aoyama, and K. Kondo, Safety of dietary conjugated linoleic acid (CLA) in a 12 week trial in healthy overweight Japanese male volunteers, Journal of Oleo Science, 56(10), 2007, 517-525.

[20] S. Benjamin, P. Prakasan, S. Sreedharan, A. Wright, and F. Spener, Pros and cons of CLA consumption: an insight from clinical evidences, Nutrition \& Metabolism, 12, 2015, 4. 